Pacific Journal of Mathematics

NIELSEN NUMBERS AND LEFSCHETZ NUMBERS ON 


\title{
NIELSEN NUMBERS AND LEFSCHETZ NUMBERS ON SOLVMANIFOLDS
}

\section{CHRISTOPHER MCCORD}

\begin{abstract}
A compact solvmanifold $S$ is a homogeneous space of a simply connected solvable Lie group: $S=\widetilde{S} / H$, with $H \subseteq \widetilde{S}$ a uniform subgroup. If $f: S \rightarrow S$ is a continuous self map on $S$, we show that $|L(f)| \leq N(f)$, where $N(f)$ is the Nielsen number of $f$ and $L(f)$ is the Lefschetz number of $f$. Necessary conditions and sufficient conditions in terms of $\pi_{1}(S)$ and $f_{\#}$ are found for the equality $N(f)=|L(f)|$ to hold.
\end{abstract}

In [3], Brooks, Brown, Pak and Taylor show that for a self map $f: M \rightarrow M$ on a torus, the Nielsen number $N(f)$ and Lefschetz number $L(f)$ are equal up to a sign, i.e. $N(f)=|L(f)|$. In [1] and [4], this result is extended to compact nilmanifolds (homogeneous spaces of nilpotent Lie groups). In this work, we examine how far these results may be extended to compact solvmanifolds (homogeneous spaces of solvable Lie groups). The equality does not hold for all self maps on solvmanifolds, as the following example of Anosov [1] shows. The Klein bottle $K$ is a compact solvmanifold, which may be represented as $\mathbb{R}^{2}$ with equivalence relation $(x, y) \sim\left(x+k,(-1)^{k} y+l\right)$ for $k, l \in \mathbb{Z}$. Define $f: K \rightarrow K$ by $f[x, y]=[-x, 2 y]$. The map has four fixed points, all in distinct fixed point classes; three have index 1 and one has index -1 . Thus $N(f)=4$ and $L(f)=2$.

Indeed, we conjecture that the only compact solvmanifolds for which $|L(f)|=N(f)$ for all maps are nilmanifolds. However, for all maps on solvmanifolds, $|L(f)| \leq N(f)$, so the Lefschetz number provides a lower bound on the fixed point set of $f$. Further, a number of conditions exist which guarantee equality of the Lefschetz and Nielsen numbers. The crucial step in establishing the inequality and the conditions for equality is the construction of a fibration for $S$. It is well known [7] that every compact solvmanifold admits a fiber bundle with a torus as the base and a compact nilmanifold as the fiber. In $\S 1$, we examine the properties of this so-called Mostow fibration. We show that every map on $S$ is homotopic to a fiber-preserving map of a 
Mostow fibration, so that the results of [1], [3] and [4] may be applied. If $S$ admits an orientable Mostow fibration, then $|L(f)|=N(f)$ for all maps. Unfortunately, such fibrations are never orientable unless $S$ is a nilmanifold.

Both the Nielsen number and Lefschetz number may be computed from two pieces of information: the number of fixed point classes, and the index of each class. In $\S 2$, we show that the number of fixed point classes is given by the number of fixed point classes in the base and in each fiber; and that each class either has index of +1 or -1 . The inequality $|L(f)| \leq N(f)$ follows easily from this, while finding necessary conditions and sufficient conditions for $N(f)=|L(f)|$ reduces to finding necessary and sufficient conditions for the index of all classes to have the same sign. A variety of such conditions are developed in $\S 3$.

1. Solvmanifolds. Mal'cev [6] has shown that any compact nilmanifold $N$ can be represented as a homogeneous space of a connected simply connected nilpotent Lie group $\widetilde{N}$, with $\widetilde{N}$ the universal cover of $N$. That is, $\pi_{1}(N)$ embeds in $\widetilde{N}$ as a discrete uniform subgroup, with $N=\tilde{N} / \pi_{1}(N)$. The corresponding statement is false for solvmanifolds: a compact solvmanifold $S$ is always a homogeneous space $\widetilde{S} / \Gamma$ of a connected simply connected solvable Lie group $\widetilde{S}$, but $\Gamma$ may not be discrete [7]. With such a representation, $\pi_{1}(S) \cong \Gamma / \Gamma_{0}$, where $\Gamma_{0}$ is the connected component of the identity of $\Gamma$.

The Lie group $\widetilde{S}$ contains a unique maximal analytic nilpotent subgroup $\widetilde{M}$, which contains $\Gamma_{0}$ and the commutator subgroup $[\widetilde{S}, \widetilde{S}]$ as normal subgroups. Thus $\widetilde{S} / \widetilde{M}$ is isomorphic to Euclidean space and $T=\widetilde{S} / \Gamma \widetilde{M}$ is a torus with fundamental group $\pi_{1}(T) \cong \Gamma / \Gamma \cap \widetilde{M}$. Similarly $N=\widetilde{M} / \Gamma \cap \widetilde{M}=\Gamma \widetilde{M} / \Gamma$ is a compact nilmanifold with fundamental group $\pi_{1}(N) \cong \Gamma \cap \widetilde{M} / \Gamma_{0}$. That is, if fiber, total space and base of the fibration $\widetilde{M} \rightarrow \widetilde{S} \rightarrow \widetilde{S} / \widetilde{M}$ are quotiented by $\Gamma \cap \widetilde{M}, \Gamma$ and $\Gamma / \Gamma \cap \widetilde{M}$ respectively, there is a resulting fibration of homogeneous spaces $N \rightarrow S \stackrel{p}{\longrightarrow} T$. Thus $S$ may be fibered over a torus with a compact nilmanifold as fiber [7].

This fibration was obtained from the analytic nilpotent subgroup $\widetilde{M}$. Clearly, a similar construction can be generated by an analytic nilpotent subgroup $\widetilde{N} \subseteq \widetilde{S}$ which contains $[\widetilde{S}, \widetilde{S}]$. We will refer to any such fibration as a Mostow fibration of $S$.

Note that in a Mostow fibration $N \rightarrow S \stackrel{p}{\longrightarrow} T$, all of the spaces are Eilenberg-Mac Lane spaces, so the homotopy exact sequence reduces 
to a short exact sequence of fundamental groups

$$
1 \rightarrow \pi_{1}(N) \rightarrow \pi_{1}(S) \stackrel{p_{\#}}{\longrightarrow} \pi_{1}(T) \rightarrow 0 .
$$

That is, $S$ is an Eilenberg-Mac Lane space with solvable fundamental group, and $\pi_{1}(N)$ is a normal nilpotent subgroup of $\pi_{1}(S)$ with $\pi_{1}(S) / \pi_{1}(N)$ free abelian.

We now consider the structure of the fundamental group $\pi=\pi_{1}(S)$. $\pi$ is a discrete torsion-free solvable group. As such, it has a unique maximal nilpotent subgroup $M_{0}$, which contains $[\pi, \pi]$. Note that a subgroup $H$ is normal, nilpotent and has $\pi / H$ abelian if and only if $[\pi, \pi] \subseteq H \subseteq M_{0}$. We will be interested in finding subgroups $H$ of this type for which the exact sequence $1 \rightarrow H \rightarrow \pi \rightarrow \pi / H \rightarrow 0$ represents the fundamental group sequence of a Mostow fibration. Let $\mathcal{N}(\pi)=\left\{H \subseteq \pi:[\pi, \pi] \subseteq H \subseteq M_{0}, \pi / H\right.$ is torsion free $\}$. For any subgroup $H$ with $[\pi, \pi] \subseteq H \subseteq M$, there is a unique minimal subgroup $C(H) \in \mathscr{N}(\pi)$ with $H \subseteq C(H)$. Further, $\mathscr{N}(\pi)$ has unique minimal element $C=C([\pi, \pi])$ and unique maximal element $M$.

THEOREM 1.1. Given a compact solvmanifold $S$ with $\pi_{1}(S)=\pi$ and $H \subseteq \pi$, there exists a Mostow fibration $N \rightarrow S \rightarrow T$ with $\pi_{1}(N)=$ $H$ if and only if $H \in \mathscr{N}(\pi)$. Given compact solvmanifolds $S_{1}$ and $S_{2}$ with fundamental groups $\pi_{1}\left(S_{1}\right)=\pi_{i}, H_{i} \in \mathcal{N}\left(\pi_{i}\right)$, and $f: S_{1} \rightarrow$ $S_{2}, f$ is homotopic to a fiber-preserving map of the Mostow fibrations induced by $H_{1}$ and $H_{2}$ if and only if $f_{\#}\left(H_{1}\right) \subseteq H_{2}$.

Proof. It suffices to prove the first statement for $H=M$, the maximal element of $\mathscr{N}(\pi)$. If $S=\widetilde{S} / \Gamma$ and the maximal analytic nilpotent subgroup $\widetilde{M} \subseteq \widetilde{S}$ has $\pi_{1}(\widetilde{M} / \widetilde{M} \cap \Gamma)=M$, then any $H \in \mathscr{N}(\pi)$ embeds in $\widetilde{M} / \Gamma_{0}$ as a discrete torsion-free subgroup. $H$ then has an extension to an analytic subgroup of $\widetilde{M} / \Gamma_{0}$, which lifts to an analytic subgroup $\tilde{N}$ of $\widetilde{M}$. This $\widetilde{N}$ and the corresponding nilmanifold $\widetilde{N} / \widetilde{N} \cap \Gamma$ are the required spaces. To show that $N=\widetilde{M} / \widetilde{M} \cap \Gamma$ has $\pi_{1}(N)=M$, we consider one construction of $\widetilde{S}$ from $S$.

In [8], Wang shows that $\pi$ may be embedded as a closed uniform subgroup of a solvable Lie group $\widetilde{G}$ which is simply connected and which has only finitely many components. It is clear from the construction that $\widetilde{G}$ may be chosen so that $M \subseteq \widetilde{N}$, the maximal analytic nilpotent normal subgroup of $\widetilde{G}$. Auslander [2] then shows that the identity component $\widetilde{G}_{0}$ of $\widetilde{G}$ can be covered by a connected simply connected solvable Lie group $\widetilde{S}$. In this cover, the maximal 
analytic nilpotent subgroup $\widetilde{M}$ of $\widetilde{S}$ maps onto $\tilde{N}$ and the subgroup $\Gamma$ which maps onto $\pi$ has $\widetilde{S} / \Gamma$ compact and $\pi_{1}(\tilde{S} / \Gamma) \cong \pi$. Since compact solvmanifolds with isomorphic fundamental groups are homeomorphic [7], $\widetilde{S} / \Gamma=S$. Further, $\widetilde{M} \cap \Gamma$ maps onto $M$, so $\pi_{1}(\widetilde{M} / \widetilde{M} \cap \Gamma)=M$.

To prove the second assertion, let $N_{i} \rightarrow S_{i} \rightarrow T_{i}$ be the Mostow fibration induced by $H_{i}$. As $f_{\#}\left(H_{1}\right) \subseteq H_{2}$, there is commutative diagram

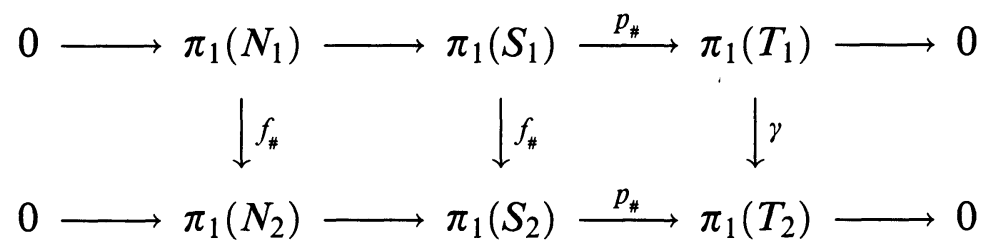

The homomorphism $\gamma: \mathbb{Z}^{a_{1}} \rightarrow \mathbb{Z}^{a_{2}}$ extends by linearity to $\Phi: \mathbb{R}^{a_{1}} \rightarrow$ $\mathbb{R}^{a_{2}}$, and so defines a quotient map $\phi: T_{1} \rightarrow T_{2}$, with $\phi_{\#}=\gamma$ on $\pi_{1}\left(T_{1}\right)$.

Since $p_{\#} \circ f_{\#}=\phi_{\#} \circ p_{\#}$, the maps $p \circ f$ and $\phi \circ p$ are homotopycommutative. Let $H: S_{1} \times I \rightarrow T_{2}$ be the homotopy with $H_{0}=p \circ f$ and $H_{1}=\phi \circ p$. Then the covering homotopy theorem implies that there is a lift $F: S_{1} \times I \rightarrow S_{2}$ of $H$ with $F_{0}=f$, so the map $F_{1}: S_{1} \rightarrow$ $S_{2}$ has $F_{1} \simeq f$ and $p \circ F_{1}=\phi \circ p$.

Note that the same proof shows that any two fiber-preserving maps which are homotopic as maps are homotopic as fiber-preserving maps.

Thus a map $f: S_{1} \rightarrow S_{2}$ may be identified as a fiber-preserving map (up to homotopy) by its behavior on the fundamental group. Suppose $S_{1}, S_{2}$ have fundamental groups $\pi_{i}=\pi\left(S_{i}\right)$ and $H_{i} \subseteq \pi_{i}$ are subgroups so that $C\left(H_{i}\right)$ is defined. If $\theta: \pi_{1} \rightarrow \pi_{2}$ has $\theta\left(H_{1}\right) \subseteq$ $H_{2}$, then $\theta\left(C\left(H_{1}\right)\right) \subseteq C\left(\theta\left(H_{1}\right)\right) \subseteq C\left(H_{2}\right)$. In particular, if $\theta: \pi \rightarrow \pi$ is an endomorphism, then $\theta([\pi, \pi]) \subseteq[\pi, \pi]$ and $\theta\left(M_{0}\right) \subseteq M_{0}$. Thus $\theta(C) \subseteq C$, and $\theta(M) \subseteq M$ if $M=M_{0}$. In general, $M$ is characteristic but not necessarily invariant under all endomorphisms.

COROLlary 1.2. If $f: S \rightarrow S$ is a self map of a compact solvmanifold, then $f$ is homotopic to a fiber-preserving map of the Mostow fibration induced by the subgroup $C=C([\pi, \pi])$.

To conclude this section, we note that any Mostow fibration is nonorientable. An element $[\omega] \in \pi_{1}(T)$ acts orientably on $N$ if 
the induced map $\tau_{\omega}: N \rightarrow N$ is homotopic to the identity. Let $O(N) \subseteq \pi_{1}(T)$ be the subgroup of elements which act orientably on $N$, so that the fibration is orientable if $O(N)=\pi_{1}(T)$.

LEMMA 1.3. If $N \rightarrow S \stackrel{p}{\longrightarrow} T$ is a Mostow fibration of $S$ with $\pi_{1}(S)=\pi$ and $\pi_{1}(N)=H \in \mathcal{N}(\pi)$, then $O(N)=p_{\#}\left(H \cdot \mathbb{C}_{\pi}(H)\right)$. $H \cdot \mathbb{C}_{\pi}(H)$ is a normal nilpotent subgroup of $\pi$, and $O(N)$ is a proper subgroup of $\pi_{1}(T)$.

Proof. The action of $\pi_{1}(T)$ on $N$ induces an action on $H$ which can be represented by conjugation of $H$ by elements of $\pi$. That is, if $d \in \pi$ and $h \in H$, then $p_{\#}(d) \cdot h=h^{d} \in H$. As $N$ is an EilenbergMac Lane space, an element $p_{\#}(d) \in \pi_{1}(T)$ acts orientably on $N$ if and only if it acts trivially on $H$. This is equivalent to requiring the action on $H$ to be conjugation in $H$. Thus $p_{\#}(d)$ acts trivially if and only if $d \in \mathbb{C}_{\pi}(H) \cdot H$ (where $\mathbb{C}_{\pi}(H)$ is the centralizer of $H$ in $\pi$ ).

If $G^{(n)}$ is the $n$th derived group of $G$, then $\left(\mathbb{C}_{\pi}(H) \cdot H\right)^{(1)} \subseteq \pi^{(1)} \subseteq$ $H^{(0)}$, and inductively, $\left(\mathbb{C}_{\pi}(H) \cdot H\right)^{(n)} \subseteq H^{(n-1)} \cdot \mathbb{C}_{\pi}(H) \cdot H$ is then nilpotent, and $O(N)=p_{\#}\left(\mathbb{C}_{\pi}(H) \cdot H\right) \subseteq p_{\#}\left(M_{0}\right) \neq p_{\#}\left(\pi_{1}(S)\right)=\pi(T) . \square$

2. Nielsen numbers and Lefschetz numbers. We now employ the Mostow fibrations of $\S 1$ to compute Lefschetz numbers and Nielsen numbers on compact solvmanifolds. As all of the quantities involved will be homotopy invariants, we assume now that $N \rightarrow S \rightarrow T$ is a Mostow fibration of $S$ and $f: S \rightarrow S$ is a fiber-preserving map with respect to the fibration. We may further assume that the induced map $\phi: T \rightarrow T$ is a homomorphism. To define the Nielsen number of $f$, the fixed point set $\operatorname{Fix}(f)$ is partitioned as follows: $x, y \in \operatorname{Fix}(f)$ belong to the same fixed point class if there exists a path $c$ from $x$ to $y$ with $f c \simeq c$. The set of fixed point classes is denoted by $\operatorname{FPC}(f)$.

Each fixed point class $\mathbb{F} \in \operatorname{FPC}(f)$ is compact and open in $\operatorname{Fix}(f)$, and so has a fixed point index, denoted $\operatorname{index}_{S}(f, \mathbb{F})$, defined. The Lefschetz number $L(f)$ is equal to the sum over all fixed point classes of the indices, while the Nielsen number $N(f)$ is the number of fixed point classes with nonzero index. There are two cases to be considered: $L(\phi)=0$ and $L(\phi) \neq 0$. If $L(\phi)=0$, then $\phi$ (and with it $f$ ) is homotopic to a fixed point free map. If $L(\phi) \neq 0$, the following lemmas establish the structure of $\operatorname{FPC}(f)$.

LEMMA 2.1. If $L(\phi) \neq 0$, then every fixed point class $\mathbb{F} \in \operatorname{FPC}(\phi)$ contains a single point. 
Proof. $\operatorname{Fix}(\phi)$ is a subgroup of $T$, with all fixed point classes homeomorphic to each other. The fixed point class of the identity $\mathbb{F}_{0}$ can be described as follows: if $\mathbb{R}^{a}$ is the universal cover of $T$ and $\Phi: \mathbb{R}^{a} \rightarrow \mathbb{R}^{a}$ the unique linear transformation which covers $\phi$, then $\mathbb{F}_{0}$ is the projection of $\operatorname{Fix}(\Phi)$. As $\mathbb{F}_{0}$ is compact subgroup of $T$, $\operatorname{Fix}(\Phi)$ is a vector subspace with $\operatorname{Fix}(\Phi) \cap \mathbb{Z}^{a}$ a uniform subgroup. If $\operatorname{Fix}(\phi) \neq 0$, then $\operatorname{Fix}(\Phi) \cap \mathbb{Z}^{a}=\operatorname{Fix}\left(\phi_{\#}\right) \neq 0$. But Fadell and Husseini [4] show that if $L(\phi) \neq 0$, then $\operatorname{Fix}(\Phi) \cap \mathbb{Z}^{a}=0$.

Henceforth, we will identify $\operatorname{Fix}(\phi)$ and $\operatorname{FPC}(\phi)$ when $L(\phi) \neq 0$. If $b \in \operatorname{Fix}(\phi)$, let $f_{b}: N_{b} \rightarrow N_{b}$ be the restriction of $f$ to $N_{b}=$ $p^{-1}(b)$. Then $\operatorname{FPC}\left(f_{b}\right)$ denotes the equivalence classes of $\operatorname{Fix}\left(f_{b}\right)$, where $x$ and $y$ are equivalent if there exists a path $c$ from $x$ to $y$ in $N_{b}$ with $f c \simeq c$ in $N_{b}$. It is clear that if $x_{1}$ and $x_{2}$ are in the same fixed point class in $N_{b}$, then they are in the same fixed point class in $S$; and that if $y_{1}$ and $y_{2}$ are in the same fixed point class in $S$, then $p\left(y_{1}\right)$ and $p\left(y_{2}\right)$ are in the same fixed point class in $T$. That is, there is a sequence of maps

$$
\operatorname{FPC}\left(f_{b}\right) \stackrel{i_{\mathrm{FPC}}}{\longrightarrow} \operatorname{FPC}(f) \stackrel{p_{\mathrm{FPC}}}{\longrightarrow} \mathrm{FPC}(\phi) .
$$

A priori, $i_{\mathrm{FPC}}$ may not be injective and $p_{\mathrm{FPC}}$ may not be onto.

LEMMA 2.2. If $L(\phi) \neq 0$, then $i_{\mathrm{FPC}}: \operatorname{FPC}\left(f_{b}\right) \rightarrow \operatorname{FPC}(f)$ is injective for every $b \in \operatorname{Fix}(\phi)$. If $L\left(f_{b}\right) \neq 0$, then $b \in \operatorname{im}\left(p_{\mathrm{FPC}}\right)$.

Proof. From [5], Theorem 4.1.6, we know that $i_{\text {FPC }}^{-1}(\mathbb{F})=\mid \operatorname{Fix}\left(\phi_{\# b}\right)$ : $p_{\#} \operatorname{Fix}\left(f_{\# s}\right) \mid$ where $s \in \operatorname{Fix}\left(f_{b}\right)$. But if $L(\phi)=0$, then $\operatorname{Fix}\left(\phi_{\# b}\right)=0$. If $L\left(f_{b}\right) \neq 0$, then there is some $s \in \operatorname{Fix}\left(f_{b}\right)$, and the fixed point class of $s$ maps to $b$.

Corollary 2.3. If $L(\phi) \neq 0$, then $\operatorname{FPC}(f)=\bigcup_{b \in \operatorname{Fix}(\phi)} \operatorname{FPC}\left(f_{b}\right)$.

We now turn to the question of computing the fixed point index for each fixed point class of $f$. From [1] and [3], all fixed point classes in $T$ have the same index, which is either $+1,0$ or -1 . Likewise, for each $b \in \operatorname{Fix}(\phi)$ all fixed point classes in $N_{b}$ have the same index in $N_{b}$, which is either $+1,0$ or -1 .

LEMMA 2.4. Every fixed point class $\mathbb{F}$ of $f$ has $\operatorname{index}_{S}(f, \mathbb{F}) \in$ $\{+1,0,-1\}$. 
Proof. If $L(\phi)=0$, then $f$ is homotopic to a fixed point free map and $N(f)=0$. That is, every fixed point class has index 0 . If $L(\phi) \neq 0$, then every fixed point class $\mathbb{F} \in \mathrm{FPC}(f)$ is represented by a fixed point class $\mathbb{F}_{b} \in \mathrm{FPC}\left(f_{b}\right)$, with $\operatorname{index}_{N}\left(f_{b}, \mathbb{F}_{b}\right)$, index $T(\phi, b) \in$ $\{+1,0,-1\}$. Thus $\operatorname{index}_{S}(f, \mathbb{F})=\operatorname{index}_{T}(\phi, b) \cdot \operatorname{index}_{N}\left(f_{b}, \mathbb{F}_{b}\right)=$ \pm 1 .

Our main result now follows immediately from 2.2 and 2.4.

THEOREM 2.5. If $S$ is a compact solvmanifold and $f: S \rightarrow S$ is a self map of $S$ then $|L(f)| \leq N(f)$. If $f$ is a fiber-preserving map of a Mostow fibration $N \rightarrow S \rightarrow T$, then $|L(f)|=N(f)$ if and only if either $L(\phi)=0$ or $\operatorname{sgn}\left(L\left(f_{b}\right)\right)$ is independent of $b \in \operatorname{Fix}(\phi)$. Further,

$$
|L(f)|=\left|L(\bar{f}) L\left(f_{b}\right)\right|=N(\bar{f}) N\left(f_{b}\right)=N(f)
$$

if and only if either $L(\phi)=0$ or $L\left(F_{b}\right)$ is independent of $b \in \operatorname{Fix}(\phi)$.

Proof. Every map is homotopic to a fiber-preserving map with respect to a Mostow fibration, with the induced map on $T$ a homomorphism $\phi$. If $L(\phi)=0$. Then $\phi$ (and hence $f$ ) is homotopic to a fixed point free map, so $L(f)=L(\phi) \cdot L\left(f_{b}\right)=N(\phi) \cdot N\left(f_{b}\right)=N(f)=0$. If $L(\phi) \neq 0, \operatorname{Fix}(\phi)$ consists of $N(\phi)$ fixed points, all of the same index \pm 1 . Then

$$
N(f)=\sum_{b \in \operatorname{Fix}(\phi)} N\left(f_{b}\right)=\sum_{b \in \operatorname{Fix}(\phi)}\left|L\left(f_{b}\right)\right| \geq\left|\sum_{b \in \operatorname{Fix}(\phi)} L\left(f_{b}\right)\right|=|L(f)| .
$$

The inequality is an equality if and only if all $L\left(f_{b}\right)$ have the same sign. If $L\left(f_{b}\right)$ is independent of $b$, then $\sum_{b \in \operatorname{Fix}(\phi)} N\left(f_{b}\right)=N(\phi) N\left(f_{b}\right)$ and $\sum_{b \in \mathrm{Fix}(\phi)}\left|L\left(f_{b}\right)\right|=\left|L(\phi) L\left(f_{b}\right)\right|$.

Note that the equality $|L(f)|=N(F)$ can occur without the product formula $|L(f)|=\left|L(\bar{f}) L\left(f_{b}\right)\right|=N(\bar{f}) N\left(f_{b}\right)=N(f)$ holding. For example the map $g: K \rightarrow K$ on the Klein bottle given by $g(x, y)=$ $(-x, y)$ covers the homomorphism $\gamma(x)=-x$ on $S^{1} \cdot \gamma$ has two fixed points +1 and -1 , each with index -1 . The fiber over each point is likewise a circle, with $L\left(g_{+1}\right)=0$ and $L\left(g_{-1}\right)=-1$. Thus $L\left(g_{+1}\right) \neq L\left(g_{-1}\right)$, but $|L(g)|=N(g)=2$. Similarly, the product formula can hold without all $f_{b}$ having the same homotopy type.

3. Conditions for equality. Suppose $f$ has the homotopy type of a fiber-preserving map of the Mostow fibration $N \rightarrow S \rightarrow T$. We now 
consider when all $b \in \operatorname{Fix}(\phi)$ have the same $\operatorname{sgn}\left(L\left(f_{b}\right)\right)$, the same $L\left(f_{b}\right)$, or the same homotopy type. Choose $b_{0}, b_{1} \in \operatorname{Fix}(\phi)$. As the fiber $N$ is a $K(\pi, 1)$, the maps $f_{0}: N_{0} \rightarrow N_{0}$ and $f_{1}: N_{1} \rightarrow N_{1}$ may be compared by comparing the induced maps $f_{i \#}: \pi_{1}\left(N_{i}\right) \rightarrow \pi_{1}\left(N_{i}\right)$. In particular, $f_{0}$ and $f_{1}$ have the same homotopy type if and only if $f_{0 \#}$ and $f_{1 \#}$ are conjugate.

If $c$ is a path in $T$ from $b_{0}$ to $b_{1}$, then there is fiber translation map $\tau_{c}: N_{0} \rightarrow N_{1}$ and commutative diagram

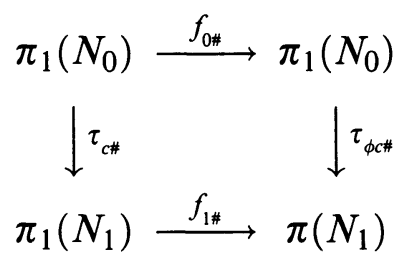

That is,

$$
\begin{aligned}
f_{1 \#} & =\tau_{\phi c \#} \circ f_{0 \#} \circ\left(\tau_{c \#}\right)^{-1}=\tau_{\phi c \#} \circ f_{0 \#} \circ\left(\tau_{c \#}\right)^{-1} \circ \tau_{\phi c \#} \circ\left(\tau_{\phi c \#}\right)^{-1} \\
& =\tau_{\phi c \#} \circ f_{0 \#} \circ\left(\tau_{\phi c \circ c^{-1}}\right)_{\#} \circ\left(\tau_{\phi c \#}\right)^{-1},
\end{aligned}
$$

so that $f_{1 \#}$ is conjugate to $f_{0 \#} \circ\left(\tau_{\phi c \circ c^{-1}}\right)_{\#}$. Fix $b_{0}=0$ and define

$$
\begin{array}{r}
\mathscr{F}(\phi)=\left\{\left[\phi c \circ c^{-1}\right] \in \pi_{1}(T, 0) \mid c: I \rightarrow T \text { with } c(0)=0,\right. \\
c(1) \in \operatorname{Fix}(\phi)\} .
\end{array}
$$

We can then compute the fiber-preserving maps from $\phi, f_{0 \#}$ and the action of $\mathscr{F}(\phi)$ on $\pi_{1}\left(N_{0}\right)$. Note that $\mathscr{F}(\phi)$ provides a simple description of $\operatorname{Fix}(\phi)$, and is itself easily computed.

LEMMA 3.1. $\mathscr{F}(\phi)$ is a subgroup of $\pi_{1}(T, 0)$, and there exists an endomorphism $\rho: \mathscr{F}(\phi) \rightarrow \operatorname{FPC}(\phi)$ given by $\rho\left(\left[\phi c \circ c^{-1}\right]\right)=c(1)$. If $\pi_{1}(T, 0)$ is embedded as the integer lattice $\mathbb{Z}^{n}$ in $\mathbb{R}^{n}$ and $\Phi: \mathbb{R}^{n} \rightarrow \mathbb{R}^{n}$ is the unique linear transformation covering $\phi: T \rightarrow T$, then $\mathscr{F}(\phi)$ embeds as $\operatorname{im}(\Phi-I) \cap \mathbb{Z}^{n}$ and $\operatorname{ker}(\rho)$ embeds as $(\Phi-I)\left(\mathbb{Z}^{n}\right)$.

Proof. If $\omega$ is a path based at 0 and $\alpha$ is a loop based at 0 , then $\omega \circ(\omega(1)+\alpha) \simeq \alpha \circ \omega$. In particular, if $c_{0}, c_{1}$ are paths from 0 to $b_{0}, b_{1} \in \operatorname{Fix}(\phi)$, then $c_{2}=c_{0} \circ\left(b_{0}+c_{1}\right)$ is a path from 0 to $b_{0}+b_{1}$, with

$$
\begin{aligned}
\phi c_{1} \circ c_{2}^{-1} & =\phi c_{0} \circ\left(b_{0}+\phi c_{1}\right) \circ\left(b_{0}+c_{1}^{-1}\right) \circ\left(c_{0}^{-1}\right) \\
& \simeq \phi c_{0} \circ\left(c_{0}^{-1}\right) \circ \phi c_{1} \circ c_{1}^{-1}
\end{aligned}
$$


and $c_{0}^{-1}-b_{0}$ is a path from 0 to $-b_{0}$ with

$$
\begin{gathered}
\phi c_{0} \circ c_{0}^{-1} \circ\left(\left(\phi c_{0}\right)^{-1}-b_{0}\right) \circ\left(c_{0}-b_{0}\right) \\
\simeq \phi c_{0} \circ\left(\phi c_{0}\right)^{-1} \circ c_{0} \circ c_{0}^{-1} \simeq \overline{0}
\end{gathered}
$$

$\rho$ will clearly be an epimorphism if it is well defined. If $c_{0}, c_{1}$ are paths from 0 to $b_{0}, b_{1} \in \operatorname{Fix}(\phi)$ such that $h: \phi c_{0} \circ c_{0}^{-1} \simeq \phi c_{1} \circ c_{1}^{-1}$, then $\omega=c_{0}^{-1} \circ c_{1}$ has $\phi \omega \simeq \omega$. Thus $b_{0}$ and $b_{1}$ are in the same fixed point class.

If $c$ is a path in $T$ from 0 to $b \in \operatorname{Fix}(\phi)$, let $c$ be the lift of $c$ based at $\mathbf{0} \in \mathbb{R}^{n}$, and let $\mathbf{x}=C(1)$. Then $\phi c$ lifts to $\Phi C$ and $\phi c \circ c^{-1}$ lifts to $\Phi C \circ\left(\Phi \mathbf{x}+C^{-1}\right)$, so $\left[\phi c \circ c^{-1}\right]$ embeds as $\Phi \mathbf{x}-\mathbf{x} \in \mathbb{Z}^{n}$. Conversely, if $m \in \operatorname{im}(\Phi-I) \cap \mathbb{Z}^{n}$, with $(\Phi-I) \mathbf{x}=\mathbf{m}$, choose a path $C$ in $\mathbb{R}^{n}$ from $\mathbf{0}$ to $\mathbf{x}$. Then $C$ projects in $T$ to a path $c$ from 0 to $b \in \operatorname{Fix}(\phi)$, and $\Phi C \circ\left(\Phi \mathbf{x}+C^{-1}\right)$ projects to $\phi c \circ c^{-1}$. But $\Phi C \circ\left(\Phi \mathbf{x}+C^{-1}\right)(1)=(\Phi-I) \mathbf{x}=\mathbf{m}$, so $\phi c \circ c^{-1}$ lifts to $\mathbf{m}$. Finally, if $\left[\phi c \circ c^{-1}\right] \in \operatorname{ker}(\rho), c$ may be chosen to be a loop at 0 . Its lift $C$ then has $C(1) \in \mathbb{Z}^{n}$, so $\left[\phi c \circ c^{-1}\right]$ embeds as $(\Phi-I) C(1) \in(\Phi-I)\left(\mathbb{Z}^{n}\right) . \square$

Note that if $L(\phi)=\operatorname{det}(\Phi-I) \neq 0$, then $\mathscr{F}(\phi)=\pi_{1}(T)$ and $\operatorname{ker}(\rho)=\operatorname{im}\left(\phi_{\#}-\mathrm{id}\right)$.

Any map $f: N_{0} \rightarrow N_{0}$ is homotopic to a homomorphism $g$, which lifts to a homomorphism $G: \widetilde{N} \rightarrow \widetilde{N}$ and so defines $D G: \mathfrak{n} \rightarrow \mathfrak{n}$, where $\mathfrak{n}$ is the Lie algebra of $\tilde{N}$. Then $L(f)=\operatorname{det}(D G-I)$. Likewise $L(\phi)=\operatorname{det}(\Phi-I)$, where $\Phi$ is the unique linear transformation on $\mathbb{R}^{n}$ covering $\phi$. Let $F: \mathfrak{n} \rightarrow \mathfrak{n}$ be the map induced from $f_{0}$ and $T_{\omega}$ the automorphism induced from $\tau_{\omega}$.

THEOREM 3.2. Suppose $f: S \rightarrow S$ is a fiber-preserving map of a Mostow fibration $N \rightarrow S \rightarrow T$ which covers homomorphism $\phi: T \rightarrow$ $T$. Then

(i) $N(f)=|L(f)|$ if and only if either $\operatorname{det}(\Phi-I)=0$ or

$$
\operatorname{sgn}\left(\operatorname{det}\left(D T_{\omega} \circ F-I\right)\right)=\operatorname{sgn}(\operatorname{det}(F-I))
$$

for all $[\omega] \in \pi_{1}(T)$;

(ii) $N(f)=N(\phi) N\left(f_{0}\right)=|L(\phi)| L\left(f_{0}\right)|=| L(f) \mid$ if and only if either $\operatorname{det}(\Phi-I)=0$ or $\operatorname{det}\left(D T_{\omega} \circ F-I\right)=\operatorname{det}(F-I)$ for all $[\omega] \in \pi_{1}(T)$;

(iii) The maps $f_{b}$ have the same homotopy type for all $b \in \operatorname{Fix}(\phi)$ if there exists $\mathscr{O} \subseteq O(N)$ such that $\mathscr{O}$ and $\operatorname{ker}(\rho)$ generate $\mathscr{F}(\phi)$. 
Proof. The only statement requiring comment is the last. The existence of such an $\mathscr{O}$ in $L(N)$ is equivalent to $O(N) \cap \mathscr{F}(\phi)$ mapping onto $\operatorname{Fix}(\phi)$ via $\rho$. If so, then for every $b \in \operatorname{Fix}(\phi)$ there exists a path $c$ from 0 to $b$ with $\left[\bar{f} c \circ c^{-1}\right] \in O(N)$, so that $\tau_{\left(\phi c \circ c^{-1}\right) \#}$ acts trivially on $\pi_{1}\left(N_{0}, 0\right)$ and $f_{0}$ is conjugate to $\tau_{\left(\phi c \circ c^{-1}\right) \#} \circ f_{b}=f_{b}$.

Having found conditions in terms of a given Mostow fibration for equality of the Lefschetz and Nielsen numbers and for the product formulas to hold, we now consider how these conditions compare for different Mostow fibrations.

LemMA 3.3. Suppose $H_{1}, H_{2} \in \mathcal{N}(\pi)$ with $f_{\#}\left(H_{i}\right) \subseteq H_{i}$ and induced homomorphism $\phi_{i}: T \rightarrow T$. Then

(i) The condition that either $L\left(\phi_{i}\right)=0$ or that the fiber maps $f_{i b}$ have the same $\operatorname{sgn}\left(L\left(f_{i b}\right)\right)$ for all $b \in \operatorname{Fix}\left(\phi_{i}\right)$ is independent of $H_{i}$.

(ii) The conditions that either $L\left(\phi_{i}\right)=0$ or that the fiber maps $f_{i b}$ have the same $L\left(f_{i b}\right)$ for all $b \in \operatorname{Fix}\left(\phi_{i}\right)$ is independent of $H_{i}$.

(iii) If $H_{1} \subseteq H_{2} \subseteq H_{1} \circ \mathbb{C}_{\pi}\left(H_{1}\right)$ and there exists $\mathscr{O}_{2} \subseteq O\left(H_{2}\right)$ such that $\mathscr{O}_{2}$ and $\operatorname{ker}\left(\rho_{2}\right)$ generate $\mathscr{F}\left(\phi_{2}\right)$, then there exists $\mathscr{O}_{1} \subseteq O\left(H_{1}\right)$ such that $\mathscr{O}_{1}$ and $\operatorname{ker}\left(\rho_{1}\right)$ generate $\mathscr{F}\left(\phi_{1}\right)$.

Proof. The first condition is equivalent to $N(f)=|L(F)|$. Since this is independent of the Mostow fibration, the condition is independent of $H$.

It suffices to prove (ii) for the case $H_{1} \subseteq H_{2}$. Requiring all $f_{b}$ to have the same $L\left(f_{b}\right)$ is equivalent to requiring that all fixed point classes have the same sign, and that every $b \in \operatorname{Fix}(\phi)$ has the same number of fixed points in $S$ covering it. If this is true for $H_{1}$ and $H_{1} \subseteq H_{2}$, consider the fibration $N_{2} / N_{1} \rightarrow T_{1} \stackrel{\psi}{\longrightarrow} T_{2}$. The fibration is orientable, and the homomorphism $\phi_{1}: T_{1} \rightarrow T_{1}$ is a fiber-preserving map covering $\phi_{2}: T_{2} \rightarrow T_{2}$. Then $L\left(\phi_{1}\right)=L\left(\phi_{2}\right) L(\tilde{\phi})$ and every fixed point in $T_{2}$ is covered in $T_{1}$ by $L(\tilde{\phi})$ fixed points. But every fixed point in $T_{1}$ is covered by $L\left(f_{1 b}\right)$ fixed points, so every fixed point in $T_{2}$ is covered by $L(\tilde{\phi}) \cdot L\left(f_{1 b}\right)=L\left(f_{2 b}\right)$ fixed points.

Conversely, if the condition holds for $\mathrm{H}_{2}$, then every fixed point in $G_{2}$ is covered by $L\left(f_{2 b}\right)$ fixed points in $S$, and by $L(\tilde{\phi})$ fixed points in $T_{1}$. Thus if $L\left(f_{2 b}\right)=L\left(f_{1 b}\right) L(\tilde{\phi})$, every fixed point in $T_{1}$ is covered by $L\left(f_{1 b}\right)$ fixed points in $S$. A simple induction argument along the lines of [4] shows that $L\left(f_{2 b}\right)=L\left(f_{1 b}\right) L(\tilde{\phi})$ for any nilmanifold fibration $N_{1} \rightarrow N_{2} \rightarrow N_{2} / N_{1}$. 
For the last statement, note that $O\left(H_{2}\right) \subseteq \psi_{\#} O\left(H_{1}\right)$, and that $\mathscr{F}(\tilde{\phi})$ $\subseteq O\left(H_{1}\right)$. Also, there is a commutative diagram of exact sequences

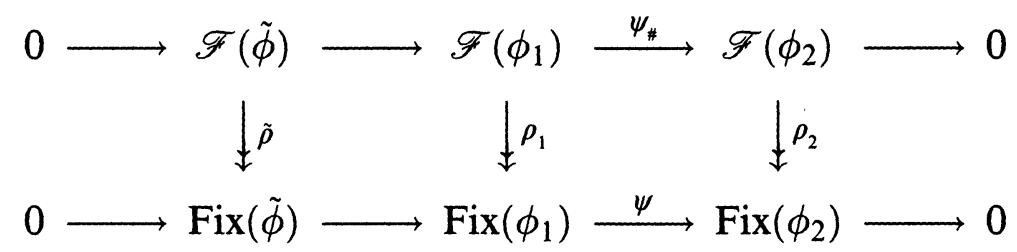

Thus, if $\mathscr{O}_{2} \subseteq O\left(H_{2}\right)$ and $\operatorname{ker}\left(\rho_{2}\right)$ generate $\mathscr{F}\left(\phi_{2}\right)$, then $\mathscr{O}_{1}=\psi^{-1}\left(\mathscr{O}_{2}\right)$ $\subseteq O\left(H_{1}\right)$ and $\operatorname{ker}\left(\rho_{1}\right)$ generate $\mathscr{F}\left(\phi_{1}\right)$.

That is, testing for equality of the Lefschetz number and Nielsen number, or for the product formula, can be done equally well with any Mostow fibration. Since every map is homotopic to a fiber-preserving map of the Mostow fibration induced by $C$, we conclude by considering the implications of this section for this fibration. Let $H_{1}(S ; \mathbb{Z})=$ $F \oplus T$, where $F$ is torsion-free and $T$ is finite. Then for the Mostow fibration of $C, \pi_{1}(T)$ is isomorphic via the Hurewicz homomorphism $h$ to $F$, which in turn embeds in $H_{1}(S ; \mathbb{R})$ by the inclusion $\imath: H_{1}(S ; \mathbb{Z}) \rightarrow H_{1}(S ; \mathbb{R})$. But $H_{1}(S ; \mathbb{R})$ is also isomorphic to the universal cover $\mathbb{R}^{n}$ of $T$, with $\Phi=f_{1 *}: H_{1}(S ; \mathbb{R}) \rightarrow H_{1}(S ; \mathbb{R})$. With these identifications, we obtain a condition for equality of the Lefschetz number and Nielsen number which requires only the computation of $O(C)$ and of $f_{1 *}: H_{1}(S ; \mathbb{R}) \rightarrow H_{1}(S ; \mathbb{R})$.

COROLlary 3.4. If $f: S \rightarrow S$ is a self map of a solvmanifold such that either $\operatorname{det}\left(f_{1 *}-\mathrm{id}\right)=0$ or $l \circ h(O(C))$ and $l \circ\left(f_{1 *}-\mathrm{id}\right) H_{1}(S ; \mathbb{Z})$ generate $\imath \circ H_{1}(S ; \mathbb{Z})$, then $N(f)=|L(f)|$ and $\operatorname{det}\left(f_{1 *}-\mathrm{id}\right)$ divides $N(f)$.

\section{REFERENCES}

[1] D. V. Anosov, The Nielsen number of maps of nil-manifolds, Russian Math. Surveys, 40 (1985), 149-150.

[2] Louis Auslander, Fundamental groups of compact solvmanifolds, Amer. J. Math., 82 (1960), 689-697.

[3] R. Brooks, R. Brown, J. Pak and D. Taylor, Nielsen numbers of maps of tori, Proc. Amer. Math. Soc., 52 (1975), 398-400.

[4] Edward Fadell and Sufian Husseini, On a theorem of Anosov on Nielsen numbers for nilmanifolds, Nonlinear Functional Analysis and its Applications (Maratea, 1985), 47-53, NATO Adv. Sci. Inst. Ser. C: Math. Phys. Sci., 173, Reidel, Dordrecht-Boston, Mass. 1986. 
[5] Jiang, Boju, Lectures on Nielsen Fixed Point Theory, Contemporary Math., 14, Amer. Math. Soc., Providence, R.I., 1983.

[6] A. I. Mal'cev, On a class of homogeneous spaces, Amer. Math. Soc. Transl., 39 (1951), 276-307.

[7] G. D. Mostow, Factor spaces for solvable groups. Ann. of Math., 60 (1954), 1-27.

[8] Hsien-Chung Wang, Discrete subgroups of solvable Lie groups I, Ann. of Math., 64 (1956), 1-19.

Received February 21, 1989 and in revised form July 3, 1989. Work supported in part by DARPA Applied and Computational Mathematics Program.

UNIVERSITY OF CINCINNATI

Cincinnati, OH 45221 


\section{PACIFIC JOURNAL OF MATHEMATICS EDITORS}

V. S. VARADARAJAN

(Managing Editor)

University of California

Los Angeles, CA 90024-1555-05

Herbert Clemens

University of Utah

Salt Lake City, UT 84112

THOMAS ENRIGHT

University of California, San Diego

La Jolla, CA 92093

\section{R. FINN}

Stanford University

Stanford, CA 94305

HermanN FlaschKa

University of Arizona

Tucson, AZ 85721

VAUGHAN F. R. JoNES

University of California

Berkeley, CA 94720

STEVEn KeRCKHOFF

Stanford University

Stanford, CA 94305
C. C. MOORE

University of California

Berkeley, CA 94720

MARTIN SCHARLEMANN

University of California

Santa Barbara, CA 93106

Harold STARK

University of California, San Diego La Jolla, CA 92093

\section{ASSOCIATE EDITORS}

R. ARENS

E. F. BECKENBACH (1906-1982)
B. H. NeumanN

\section{SUPI}

UNIVERSITY OF ARIZONA

UNIVERSITY OF BRITISH COLUMBIA

CALIFORNIA INSTITUTE OF TECHNOLOGY

UNIVERSITY OF CALIFORNIA

MONTANA STATE UNIVERSITY

UNIVERSITY OF NEVADA, RENO

NEW MEXICO STATE UNIVERSITY

OREGON STATE UNIVERSITY
F. WolF
K. YoshidA
(1904-1989)

UNIVERSITY OF OREGON UNIVERSITY OF SOUTHERN CALIFORNIA

STANFORD UNIVERSITY

UNIVERSITY OF HAWAII

UNIVERSITY OF TOKYO

UNIVERSITY OF UTAH

WASHINGTON STATE UNIVERSITY

UNIVERSITY OF WASHINGTON 


\section{Pacific Journal of Mathematics}

Vol. 147, No. $1 \quad$ January, 1991

Mark S. Ashbaugh, Evans Malott Harrell, II and Roman Svirsky, On minimal and maximal eigenvalue gaps and their causes $\ldots \ldots \ldots \ldots \ldots 1$

Robert Coleman and Francis Oisin McGuinness, Rational formal group

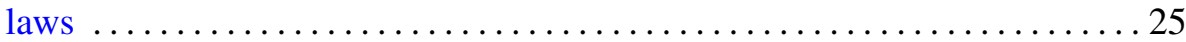

Jacek M. Cygan and Leonard Frederick Richardson, $D$-harmonic distributions and global hypoellipticity on nilmanifolds ...........29

Satya Deo and Kalathoor Varadarajan, Some examples of nontaut

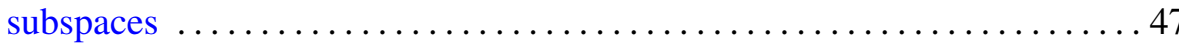

Maria Fragoulopoulou, Automatic continuity of *-morphisms between

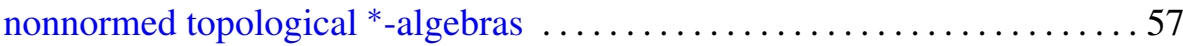

Stephen J. Gardiner, Removable singularities for subharmonic functions . . . 71 Herbert Paul Halpern, Victor Kaftal and László Zsidó, Finite weight projections in von Neumann algebras $\ldots \ldots \ldots \ldots \ldots \ldots \ldots \ldots \ldots \ldots$

Telemachos E. Hatziafratis, Explicit $\bar{\partial}$-primitives of Henkin-Leiterer kernels on Stein manifolds

Ka Hin Leung, A construction of an ordered division ring with a rank one

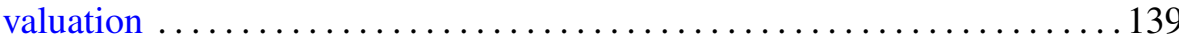

Christopher K. McCord, Nielsen numbers and Lefschetz numbers on solvmanifolds

Katsuro Sakai and Raymond Y. T. Wong, Manifold subgroups of the homeomorphism group of a compact $Q$-manifold

Caroline Perkins Sweezy, $L$-harmonic functions and the exponential square class 